\title{
Icterícia neonatal e os cuidados de enfermagem: relato de caso
}

\author{
Fernanda Thais Silva Carvalho ${ }^{1}$ \\ Mariana Viana Almeida ${ }^{2}$
}

\begin{abstract}
${ }^{1}$ Enfermeira pela Universidade Paulista e Residente em Enfermagem Obstétrica pela SES/DF
${ }^{2}$ Enfermeira Obstetra e Tutora do Programa de Residência Uniprofissional em Enfermagem Obstétrica da ESCS/ FEPECS/SES-DF
\end{abstract}

Email para correspondência: fethaiscarvalho@gmail.com

\section{RESUMO}

$\mathrm{Na}$ icterícia neonatal tem uma hiperbilirrubinemia em geral indireta que decorre do acúmulo do pigmento bilirrubínico não conjugado levando ao aparecimento da coloração amarelada de pele e mucosas. Grande parte dos recém-nascidos desenvolvem essa condição clínica na primeira semana de vida devido ao processo adaptativo do neonato ao metabolismo da bilirrubina. Embora curse de forma benigna em sua grande maioria, em alguns casos, a bilirrubina indireta, por ser lipossolúvel, pode impregnar no sistema nervoso central e causar a encefalopatia bilirrubínica que em sua fase crônica pode ocasionar paralisia cerebral e deficiência mental. Dessa forma, por ser prevalente e levando em consideração o risco de gravidade, o presente relato de caso buscou descrever a icterícia neonatal, suas repercussões no binômio mãe-bebê e a assistência de enfermagem frente ao caso.

Palavras-chave: Icterícia Neonatal; Hiperbilirrubinemia; Binômio; Assistência de Enfermagem.

\section{Neonatal jaundice and nursing care: case report}

\begin{abstract}
In neonatal jaundice, there is a general indirect hyperbilirubinemia that results from the unconjugated bilirubin pigment that leads to the yellowish coloration of the skin and mucous membranes. Most newborns develop this clinical condition in the first week of life due to the newborn's adaptive process to bilirubin metabolism. Although the course is benign in its great majority, an indirect bilirubin can impregnate the central nervous system and cause a bilirubin encephalopathy that in its chronic phase can cause cerebral and mental paralysis. Thus, because it is prevalent and considers the risk of severity, the present case will describe neonatal jaundice and its repercussions on the mother-baby binomial and nursing care.
\end{abstract}

Keywords: Neonatal Jaundice; Hyperbilirubinemia; Binomial; Nursing Assistance. 


\section{INTRODUÇÃO}

A icterícia neonatal decorre do acúmulo do pigmento bilirrubínico não conjugado, sendo que cerca de 80 a $90 \%$ da bilirrubina é proveniente da quebra da hemoglobina ou de eritropoiese ineficaz. Em suma, a bilirrubina é produto do catabolismo do heme. A enzima heme oxigenase, catalisa a quebra do heme resultando na biliverdina que rapidamente se transforma em bilirrubina indireta por ação da biliverdina redutase, esta, liga-se a albumina para ser transportada para o fígado, e ao chegarem ao hepatócito se desassociam-se para que a bilirrubina indireta seja absorvida e processada. A enzima uridina difosfogluconurato glucuronosiltransferase (UGT1A1) promove a conjugação da bilirrubina com o ácido glucorônico produzindo a bilirrubina direta, que por ser hidrossolúvel, será mais facilmente excretada pelo sistema biliar e trato gastrointestinal ${ }^{1,2}$.

A icterícia se manifesta clinicamente quando os níveis séricos de bilirrubina se elevam a valores $>5 \mathrm{mg} / \mathrm{dl}^{3,4}$. A suscetibilidade do $\mathrm{RN}$ para desenvolvê-la está associado a um maior volume eritrocitário (valores de hematócrito entre 50 a 60\%); menor meia vida das hemácias (aproximadamente 85 dias); menor capacidade de conjugação e excreção, principalmente pela deficiência da enzima UGT1A1 (glucuronosiltransferase) e por possuir trato gastrointestinal estéril (aproximadamente 85 dias), o que permite um aumento da desconjugação na mucosa intestinal ${ }^{2}$. O pico de bilirrubina e o tempo de resolução dependem de algumas variáveis: dieta, idade gestacional e etnia ${ }^{2}$.

\section{RELATO DE CASO}

Paciente do sexo feminino, $3^{\circ}$ dia de vida, pesando $3050 \mathrm{~g}$, recém nascida a termo (Idade Gestacional de 37 semanas e 6 dias), pós parto normal induzido por SHEG (Síndrome Hipertensiva Específica Gestacional), bolsa rota de aproximadamente 7 horas, com líquido amniótico claro, apresentação cefálica, clampeamento precoce, não 
necessitou manobras de reanimação, com peso ao nascer de $3.360 \mathrm{~g}$, Apgar 9 no $1^{\circ}$ minuto e 10 no $5^{\circ}$ minuto, estatura de $49 \mathrm{~cm}$. Tipo sanguíneo da criança: B Rh+, Coombs direto negativo. Puérpera, G1Pn1A0, realizou pré-natal com 6 consultas, sorologias não reagentes, glicemia sem alterações, com uso de metildopa para controle de níveis pressóricos. Tipo sanguíneo da mãe: $\mathrm{O} R \mathrm{Rh}+$. Com 72 horas de vida, colhido BT e F (Bilirrubina Total e Frações) do neonato cujos valores foram: Bilirrubina Total: 14,44 $\mathrm{mg} / \mathrm{dl}$; direta: 0,27 mg/dl e indireta: $14,17 \mathrm{mg} / \mathrm{dl}$. Conduta médica frente aos resultados: indicada fototerapia contínua. Mãe apresenta-se ansiosa e preocupada em relação à fototerapia e a saúde do bebê.

\section{DISCUSSÃO}

A hiperbilirrubinemia pode decorrer da adequação ao metabolismo da bilirrubina pelo neonato (também chamada de icterícia fisiológica) ou ser advinda de processos patológicos ${ }^{1}$. Quando patológica, deve ter suas causas identificadas a fim de prevenir o desenvolvimento da hiperbilirrubinemia grave (>25mg/dl). Muitas vezes, o aparecimento da icterícia antes das primeiras 24 horas de vida pode estar associado à presença de doenças hemolíticas por incompatibilidade $\mathrm{RH}$, incompatibilidade $\mathrm{ABO}$, deficiência de G6PD e sepse ${ }^{1,2}$. Cerca de 12 a $15 \%$ dos fetos do grupo A ou B e mães do grupo O apresentam incompatibilidade $\mathrm{ABO}$, quando há uma positividade de Coombs aumentase a suspeita de doença hemolítica por incompatibilidade mãe e feto ${ }^{1,5}$.

Outras causas que culminam no aumento da produção de bilirrubina incluem a policitemia e as coleções sanguíneas extravasculares como os celafohematomas e equimoses $^{2,5}$. Fatores que aumentam a bilirrubina na circulação enterohepática incluem as anomalias gastrintestinais, uma oferta inadequada do leite materno (ingesta abaixo do ideal) e o tempo do clampeamento do cordão ${ }^{6}$. É sabido que o clampeamento tardio do 
cordão umbilical apresenta diversos benefícios à saúde do bebê, mas comumente, o aparecimento da icterícia neonatal com necessidade de tratamento é relacionado ao clampeamento tardio do cordão umbilical (prática recomendada pela Organização Mundial da Saúde), pela passagem aumentada de hemoglobina. Porém, estudos realizados sugerem que não há um aumento significativo do aparecimento da policitemia e da icterícia e o manejo desta com necessidade de tratamento, em recém-nascidos cujos cordões foram clampeados tardiamente. ${ }^{7,8,9}$.

Alguns defeitos diminuem a conjugação da bilirrubina, principalmente aqueles associados a mutações nos genes da UGT1A1, como a Síndrome de Gilbert e Crigler Najjar tipo I e II. Nesta classificação incluem-se também o hipotireoidismo congênito e a Síndrome da Icterícia pelo Leite Materno (ocasionada pelas altas concentrações da betaglicuronidase $)^{2}$. O aleitamento materno exclusivo (AME), quando ocorre de forma inadequada (por dificuldade de sucção ou pouca oferta láctea) associa-se ao desenvolvimento de hiperbilirrubinemia durante a primeira semana de vida, pois aumenta a circulação enterohepática da bilirrubina sobrecarregando o hepatócito. Essa icterícia do aleitamento pode estar acompanhada de perda de peso (>7\% em relação ao nascimento) e quadros de desidratação. Por outro lado, na Síndrome da Icterícia pelo Leite Materno, substâncias presentes no leite ativam genótipos que estão associados a menor conjugação hepática, acarretando uma icterícia mais persistente (prolongando-se por mais de 2 semanas), porém chama-se a atenção o bom estado geral do recém-nascido com adequado ganho ponderal ${ }^{6,10}$.

Além dos fatores mencionados, estudos realizados identificaram uma associação da indução do trabalho de parto com utilização da ocitocina ao aparecimento da icterícia. A explicação para esta ocorrência é que a ocitocina por possuir um efeito semelhante à vasopressina (ADH- hormônio antidiurético), causaria hiposmolaridade que poderia 
evoluir com edema nos glóbulos fetais deixando-os mais suscetíveis à hemólise. Porém, quando a ocitocina é utilizada em baixas doses, esta incidência diminui ${ }^{11,12,13}$.

No quadro clínico da icterícia, tem-se a coloração amarelada de pele e mucosas com progressão cefalocaudal, que usualmente é classificada com relação as zonas de Kramer. Cada uma das zonas se relaciona a algum nível aproximado de Bilirrubina Total (BT), sendo que uma icterícia visível nos membros pode estar relacionada a maiores valores de BT. Alguns fatores como a experiência do profissional, pigmentação da pele do bebê e a luminosidade do local podem interferir na visualização da icterícia ${ }^{6}$.

Assim como realizado no relato de caso em questão, para o diagnóstico laboratorial da icterícia, faz-se necessário a dosagem de bilirrubina total e frações (tanto a direta quanto a indireta), além da tipagem sanguínea de ambos (mãe e bebê) e a realização do Coombs direto. Além destes, outros exames podem auxiliar no diagnóstico, tais como: pesquisa de anticorpos anti-D quando mãe Rh negativo; pesquisa de anticorpos para antígenos irregulares quando mãe multigesta, com histórico anterior de transfusão sanguínea ou RN com positividade de Coombs direto; além de dosagem da glicose-6fosfato desidrogenase (G6PD) e dos hormônios tireoidianos ${ }^{3,6}$.

O Normograma de Buthani orienta a determinação do risco de hiperbilirrubinemia, levando em consideração a idade pós natal. A definição de hiperbilirrubinemia clinicamente significante apresenta divergências na literatura: de acordo com o Guia para Profissionais da Saúde elaborado pelo Ministério da Saúde em $2014^{6}$, os níveis de bilirrubina vão de 15 a 17mg/dl; porém estudo Wong em $2019^{2}$, revela valores de BT acima do percentil 95 no Normograma de Buthani. Em relação aos valores de hiperbilirrubinemia grave $(\mathrm{BT}>25 \mathrm{mg} / \mathrm{dl})$ e extrema $(\mathrm{BT}>30 \mathrm{mg} / \mathrm{dl})$, as duas literaturas apresentam consenso ${ }^{2,6}$. 
Embora curse rotineiramente com evolução benigna, a hiperbilirrubinemia indireta pode, quando excessivamente elevada, causar danos ao sistema nervoso dos recém nascidos. A maior complicação da icterícia é a encefalopatia bilirrubínica, também chamada de Kernicterus em sua fase crônica, que ocorre quando a bilirrubina, por ser lipossolúvel, atravessa a barreira hematoencefálica, podendo causar lesões principalmente aos gânglios de base ${ }^{5}$.

A evolução da encefalopatia compreende duas fases: inicialmente apresenta hipotonia, sucção débil, pode haver febre e convulsões e hipertonia manifestada pelo retroarqueamento do tronco. Esta fase pode ser reversível quando precocemente tratada. A fase crônica caracteriza-se pela paralisia cerebral, neuropatia auditiva, limitação do olhar para cima, displasia dentária e, ocasionalmente, deficiência mental ${ }^{6}$.

Com o intuito de prevenir tais agravos o diagnóstico e o tratamento devem ser precoces. O tratamento da icterícia neonatal mais comumente utilizado é a fototerapia, por ser um método não invasivo. O mecanismo de ação da fototerapia decorre de reações químicas da bilirrubina na pele quando expostas à luz. Tais reações alteram a estrutura da molécula da bilirrubina, em processos de fotoisomerização, transformando- a em produtos mais solúveis e passíveis de serem excretados pelo fígado.

Outro mecanismo de ação da fototerapia é a fotooxidação, que ocorre mais tardiamente (após 72 horas); que fragmenta a bilirrubina levando à produção de complexos mais solúveis em água para serem excretados na urina ${ }^{14,15}$. Para que a fototerapia seja eficaz, há dependência de alguns aspectos: comprimento de onda da luz; irradiância espectral, distância da luz e o recém-nascido e superfície do corporal exposta à luz ${ }^{6}$.

$\mathrm{Na}$ assistência de enfermagem, é preciso atentar-se aos cuidados em relação ao bebê quando este está sob efeito da luz. Estes cuidados envolvem a proteção de olhos com 
cobertura radiopaca (atentando-se para que os olhos estejam fechados na inserção para evitar possíveis escoriações), realização de limpeza ocular (ao menos duas vezes ao dia) com solução fisiológica; verificar a distância entre o bebê e a fonte luminosa (30 a 50 cm) afim de prevenir queimaduras, não utilizar produtos à base de óleos e pomadas; observar a frequência, quantidade e aspecto das eliminações, devido ao risco de decréscimo ou aumento exacerbado nas eliminações do RN. Para que a fototerapia seja eficiente é imprescindível que haja exposição da superfície corporal do bebê, com mudança de decúbito a cada $3 \mathrm{~h}$, visando distribuição uniforme da luz ${ }^{14,15,16,17}$.

Durante o tratamento fototerápico, o recém-nascido por estar despido e exposto a uma fonte de calor pode apresentar oscilações de temperatura, sendo essencial a realização de curva térmica a cada três (3) horas, aliada à verificação dos outros sinais vitais constantemente.

A realização de exame físico minucioso e observação clínica (a cada 8-12h) auxilia na identificação precoce de possíveis alterações, sendo importante a pesagem diária do neonato para observação de ganho ou perda ponderal. A amamentação deve ser supervisionada e apoiada de forma constante, com incentivo ao aumento na frequência das mamadas (10 a 12 vezes ao dia) e esclarecimentos quanto à pega e posição correta $^{14,15,16,17}$.

A hospitalização do recém-nascido por icterícia gera dúvidas, insegurança, medo e ansiedade à mãe, relacionadas à falta de contato visual e limitação do estabelecimento de vínculo na troca de fraldas e durante a amamentação. Dessa forma, é imprescindível atentar-se à puérpera, prestar esclarecimentos completos com intuito de tranquilizá-la, promover um ambiente acolhedor e encorajá-la a realizar o cuidado ao $\mathrm{RN}$, recebendo medidas educativas por parte da equipe de enfermagem ${ }^{18,19}$. 


\section{CONCLUSÕES}

A icterícia neonatal é uma condição clínica prevalente nas maternidades e a familiaridade desta condição pode ocasionar assistência robotizada ao recém-nascido, com inobservância das repercussões clínicas e psicossociais, tanto individuais quanto familiares. Desta forma, a assistência singular de enfermagem ao neonato com icterícia é essencial bem como novos estudos são necessários em função de divergências na literatura acerca do manejo e o tratamento desta condição clínica que não deve ser subestimada.

\section{REFERÊNCIAS}

1- Romano DR. Icterícia neonatal no recém-nascido de termo. Portugal (Porto). [dissertação de mestrado] - Universidade do Porto, 2017.

2- Wong RJ, Bhutani VK. Unconjugated hyperbilirubinemia in the newborn Pathogenesis and etiology. UpToDate, 2019. Disponível em: https://www.uptodate.com/contents/unconjugated-hyperbilirubinemia-in-thenewborn-pathogenesis-and-etiology?search=pathogenesis-and-etiology-ofunconjugated-hyperbilirubinemia-inthe \&source=search_result\&selectedTitle=1 104\&usage_type=default\&display_ rank=1. Acesso em: junho de 2020.

3- Draque CM, Almeida MFB. Sociedade Brasileira De Pediatria. Icterícia No Recém-Nascido Com Idade Gestacional > 35 Semanas. Documento Científico, 2012. Disponível em: https://www.sbp.com.br/fileadmin/user_upload/2015/02/Ictericia_sem- 
deptoNeoSBP-11nov12.pdf.\%20\%20Acesso\%20em:\%2014\%20jan.\%202020.

Acesso em: maio de 2020

4- Junior GSN, Vieira WL, Junior JAAG. Icterícia: uma doença comum entre os recém-nascidos. Brazilian Journal of health Review 2019; (2): 2343-2350.

5- Bravo GNT. Paciente neonato con ictericia por incompatibilidad feto materno por el grupo sanguíneo abo. Ecuador (Babahoyo). [trabalho de conclusão de curso] Universidad Técnica De Babahoyo, 2019

6- Brasil. Ministério da Saúde. Secretaria de Atenção à Saúde. Departamento de Ações Programáticas Estratégicas. Atenção à saúde do recém-nascido: guia para os profissionais de saúde. Ministério da Saúde, 2014; 2 ed.

7- WHO. World Health Organization. O clampeamento tardio do cordão umbilical reduz a anemia infantil. World Health Organization - ?2014. Disponível em: https://apps.who.int/iris/bitstream/handle/10665/120074/WHO_RHR_14.19_por. pdf;jsessionid=F187TP6VrthQ6oeYUW9V9hsbvArNxMWhj8D?sequence=2. Acesso em: junho de 2020.

8- Goes JF. Clampeamento tardio do cordão umbilical: estudo de coorte. Rio de Janeiro. [dissertação de mestrado] - Fundação Oswaldo Cruz, 2017.

9- Rabello, CG. et al. Existe associação entre o clampeamento oportuno do cordão umbilical e a ocorrência de icterícia na primeira semana de vida? In: Anais do Congresso Brasileiro de Enfermagem Obstétrica e Neonatal. Campo Grande (MS) CCARGC, 2018. Disponível em: $<$ https//www.even3.com.br/anais/cobeon/60911-existe-associacao-entre-oclampeamento-oportuno-do-cordao-umbilical-e-a-ocorrencia-de-ictericia-naprimeira-semana>. Acesso em: junho de 2020. 
10- UNIFESP. Universidade Federal de São Paulo- Una-SUS. [página da internet] Módulo de casos complexos. Disponível em https://www.unasus.unifesp.br/index.php/2015-09-15-23-00-34/esf-biblioteca. Acesso em: maio de 2020

11- Kabbany ZAE, Toaima TN, EL-Din MYG. Does anaesthesia in mothers during delivery affect bilirubin levels in their neonates? Korean Journal of Pediatrics 2017;60(12):385-389.

12- Souza GN, Sakita M, Lopes V, Ferreira DQ, Mohamed SH, Souza E. Métodos de indução do trabalho de parto. Femina 2013; 41 (1): 47-54

13- Gallo GC. Inducción de labor de parto como factor asociado a ictericia neonatal en el Hospital Belén de Trujillo. Peru (Trujillo). [trabalho de conclusão de curso] Universidad Privada Antenor Orrego, 2019.

14- Lopes LC, Paes IADC. Possíveis diagnósticos e intervenções da enfermagem a neonatos em fototerapia. Revista Científica da FHO Uniararas 2015;3 (2): 57-67

15- Oliveira CS, Casati OS, Fernandes JJ, Oliveira AR, Alves ED. Fototerapia, cuidados e atuação da enfermagem. UNICiências, 2011; 15 (1): 141-152

16- Bueno M, Sacai S, Toma E. Hiperbilirrubinemía neonatal: propostas de intervenções de enfermagem. Acta Paul Enf, 2003; 16 (2): 75-83

17- Febrasgo. Federação Brasileira das Associações de Ginecologia e Obstetrícia. Série Orientações e Recomendações. Comissão Nacional Especializada em Aleitamento Materno. São Paulo 2018; 6: 120p.

18- Souza JJ, Felipe AOB, Terra FS. Fototerapia: os sentimentos das mães de recémnascidos submetidos a essa terapia. Semina: Ciências Biológicas e da Saúde 2012; 33 (2): 231-240. 
19- Araujo IRB, Oliveira LLS, Santos TMMG, Moraes SDS. Assistência de enfermagem ao recém-nascido com icterícia neonatal: uma revisão integrativa. Rev Enferm UFPI, 2014; 3(1):120-124. 